ARTIGO

Recebido em: 26/02/2016

Aceito em: 10/11/2016

\title{
Tipos e usos de ferramentas de apoio a gestão do conhecimento em uma empresa de tecnologia da informação
}

\author{
Types and uses tools of support for knowledge management in a \\ company of information technology
}

\footnotetext{
Fabio CORREA (fabiocontact@gmail.com)* - Fabricio ZIVIANI (fazist@hotmail.com)** -

Flávia Braga CHINELATO (flaviachinelato@gmail.com)***

* Professor da Universidade Federal de São João Del-Rei

** Professor da FUMEC

*** Doutoranda da FUMEC; Professora do Centro Universitário UNA
}

\begin{abstract}
Resumo
Diante da relevância da gestão do conhecimento no cenário organizacional, diversas ferramentas e metodologias para a promoção da gestão do conhecimento nas organizações são identificadas no mercado e na literatura, respectivamente. Esta pesquisa buscou identificar quais tipologias de ferramentas de apoio a gestão do conhecimento são utilizadas por uma empresa de tecnologia da informação, e como esta organização mensura o resultado obtido por esta utilização. Trata-se de uma pesquisa descritiva, com abordagem qualitativa, de estudo de caso com entrevista aberta. Concluiu-se que a empresa utiliza o $50 \%$ das tipologias analisadas sem um plano de gestão do conhecimento formalizado.
\end{abstract}

Palavras-Chave: Gestão da Informação e Conhecimento. Ferramentas de Gestão do Conhecimento. Tecnologia da Informação.

\section{Abstract}

Given the importance of knowledge management in the organizational setting, various tools and methodologies for the promotion of knowledge management in organizations are identified in the market and literature, respectively. This research sought to identify which types of tools to support knowledge management are used by an information technology company, and how this organization measures the results obtained by this operation. This is a descriptive research with a qualitative approach, case study with open interview. It was concluded that the company uses $50 \%$ of the types analyzed without a knowledge management plan formalized.

Keywords: Information and Knowledge Management. Tools of Knowledge Management. Information Technology.

v. 22, n. 48, 2017. p. $27-40$

ISSN 1518-2924

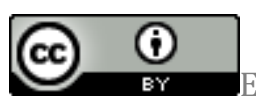

Esta obra está licenciada sob uma Licença Creative Commons. 


\section{INTRODUÇÃo}

A partir da abertura dos mercados mundiais, empresas estrangeiras passam a exercer suas atividades em outros países, orientadas por regras e políticas definidas pelos governos, acirrando a competitividade ao mercado interno e externo de atuação das organizações. Diante deste cenário a busca de vantagem competitiva perante aos concorrentes torna-se uma prática das organizações.

A vantagem competitiva é compreendida como a criação de valor pela empresa aos compradores (PORTER, 1990; PETERAF; BARNEY, 2003; BARNEY; HESTERLY, 2007) que transcende seus custos de fabricação e a diferencia em relação as outras firmas. Na era do conhecimento, este se torna o único recurso capaz de trazer um diferencial competitivo para a organização e este recurso provem das pessoas (DRUCKER, 1998; FONTANILLAS; CRUZ; GONÇALVES, 2012).

$\mathrm{Na}$ era do conhecimento, as empresas devem criar o conhecimento através da obtenção deste de fontes internas e externas à organização e armazená-lo para que seja possível sua utilização (NONAKA; TAKEUCHI, 1997), sendo as equipes de indivíduos, elementos primordiais para as organizações do futuro (KATZENBACH; SMITH, 2001) devido a suas habilidades que impulsionam as chances competitivas da firma (SPENDER, 2001).

Tão importante quanto criar o conhecimento é promover a gestão deste para que o mesmo mantenha-se dentro da organização. Para prover esta gestão, são necessárias ferramentas tecnológicas que auxiliem neste processo, atuando como interface entre o conhecimento e as ações estratégicas da empresa (VELENTIM et al, 2003), logo a tecnologia da informação é a base para realizar a captura, estruturação e transferência do conhecimento.

Inúmeras aplicações voltadas para a gestão do conhecimento (GC) são ofertadas no mercado. Carvalho (2000) sugeriu uma tipologia de 8 categorias de ferramentas tecnológicas de apoio à GC. O uso destas tecnologias apara as empresas na tomada de decisões estratégicas, mas quais tipologias de ferramentas de apoio a gestão do conhecimento são utilizadas por uma empresa de tecnologia da informação, e como esta organização mensura o resultado obtido por esta utilização?

Considerando a atuação da Empresa X no mercado de TI, a mais de 10 anos, os objetivos deste estudo são identificar a existência de uma GC, identificar as ferramentas de gestão do conhecimento utilizadas na empresa, sobre o ponto de vista da tipologia apresentada por Carvalho (2000) e como esta gestão é utilizada para a tomada de decisões pela organização.

Este estudo é alicerçado sobre os pilares gestão do conhecimento (GC) e ferramentas tecnológicas de apoio a GC. Estes elementos constituem o cerne do estudo de caso realizado e são apresentados nas seções seguintes.

\section{FUNDAMENTAÇ̃̃O TEÓRICA}

A fundamentação teórica deste estudo é baseada sobre os pilares gestão do conhecimento (GC) e ferramentas tecnológicas de apoio a GC. Estes elementos constituem o cerne do estudo de caso realizado e são apresentados nas seções seguintes.

\subsection{Gestão do Conhecimento}

Ao longo da história, as organizações passaram por mudanças processuais, tecnológicas e de mercado, causando o efeito de reverberação nas pessoas que as compõem e na sociedade. Toffler (1987) apresenta a evolução da sociedade em três ondas: agricultura, indústria e conhecimento.

A primeira onda, marcada pela atividade agrícola, a terra e mão de obra determinava o sucesso econômico. Na segunda onda os equipamentos, prédios (fábricas), matéria-prima, trabalho humano e capital financeiro tornaram-se os fatores de relevância para a indústria, enquanto na terceira onda o conhecimento torna-se o principal elemento organizacional.

A distinção destas ondas esta nos meios de geração de riqueza para as organizações. Na agrícola tem-se a terra e mão de obra, enquanto na industrial a terra perde espaço para os elementos fabris (equipamentos, prédios, outros), tornando estes obsoletos na onda do conhecimento. 
O conhecimento, uma vez sendo o elemento de maior relevância no contexto empresarial, deve ser compreendido em sua essência, que emerge de seus precedentes dado e informação.

0 dado é compreendido como quantificável, de fácil armazenagem e transferência (DAVENPORT, 1998; BASTOS; BRUNO; REZENDE, 2013), enquanto a informação são dados imbuídos de significado (DRUCKER, 1997; DAVENPORT, 1998; FERES, 2015) atribuídos por pessoas tendo característica semântica (SETZER, 1999), já o conhecimento é a transformação da informação pelas experiências do indivíduo (DAVENPORT; PRUSAK, 1998; CHOO, 2003; BASTOS; BRUNO; REZENDE, 2013; FERES, 2015).

O conhecimento assume a face tácita e explícita. 0 tácito é intangível, como crenças pessoais, valores e experiências individuais, sendo mais complexo de ser transmitido e dificilmente exprimível, enquanto o explícito é formalizado, externalizado e transmitido por documentos, manuais, dentre outros meios (NONAKA; TAKEUCHI, 1997).

Corroborando com Toffler (1987), Drucker (1998) salienta que na sociedade do conhecimento este deve ser visto como "o recurso" e não mais "um recurso". Para Davenport e Prusak (1998) o conhecimento pode ser traduzido como uma vantagem competitiva sustentável. Trata-se de um ativo "[...] para a melhoria da produtividade em um ambiente competitivo [...]" (BASTOS; BRUNO; REZENDE, 2013, p. 31)

Terra e Gordon (2002) afirmam que a principal vantagem competitiva das empresas é o capital humano traduzido pelo conhecimento tácito que seus funcionários possuem, pois este é difícil de ser copiado porque reside na cabeça das pessoas. Portanto, se o conhecimento é o elemento organizacional primordial e este advém das pessoas, sendo estas imbricadas de informação (VALENTIM, 2013), logo as pessoas “[...] são os únicos verdadeiros agentes da empresa" (SVEIBY, 1998, p. 9), sendo o alicerce das organizações.

Destaca-se que ter conhecimento não equivale a ter vantagem competitiva se este não for utilizado. De acordo com Newstrom e Pierce (2009) o objetivo do conhecimento não é o conhecimento em si, mas a ação originada a partir do uso deste.

Diante da relevância do conhecimento (tácito e explícito) no ambiente organizacional, e por sua origem ser fundamentada nas pessoas, compreende-se a GC, dentre as inúmeras definições identificadas na literatura, como um processo integrado destinado a criar, organizar, disseminar e intensificar o conhecimento, com o intuito de promover melhoria no desempenho global da organização (DAVENPORT; PRUSAK, 1998).

Desde a compreensão da importância do conhecimento no contexto organizacional, diversas propostas para a GC são apresentadas por autores elucidando modelos que tendem ao atingimento deste desafio. Para citar alguns, tem-se Nonaka e Takeuchi (1997) com a espiral do conhecimento organizacional; Davenport e Prusak (1998) com as três fases da GC; Terra (2001) com o modelo das sete dimensões; Choo (2003) com as arenas do conhecimento, Corrêa (2014) com modelo de gestão do conhecimento ao setor de TI, dentre outros.

Não basta criar o conhecimento, é necessário compartilhar este para as pessoas e torná-lo disponível na organização, ou seja, o conhecimento deve ser gerido e para prover esta gestão, são necessárias ferramentas tecnológicas que auxiliem neste processo, logo a tecnologia da informação é imprescindível neste cenário.

\subsection{Ferramentas tecnológicas de apoio a Gestão do Conhecimento}

Estudos de ferramentas, tecnológicas ou metodológicas, que apoiam a GC são elucidadas na literatura como os mapas de conhecimento, apresentados por Davenport e Prusak (1998) e Probst, Raub e Romhardt (2002), a intranet por Newell, Scarrbrough e Swan (2001) e Stenmark (2002), o Manual APO de GC (YOUNG, 2010), dentre outros.

Em relação as ferramentas tecnológicas, diversos softwares de ou apoio a GC são disponibilizados no mercado. Não é o objetivo apresentar estas ferramentas uma vez que a evolução da tecnologia é algo constante e este estudo tornar-se-á obsoleto com o tempo, mas torna-se indispensável a apresentação do estudo das tipologias destas ferramentas realizado por Carvalho (2000), para entendimento da proposição deste estudo.

Especificamente, o estudo de Carvalho (2000) apresenta tipos de ferramentas tecnológicas que apoiam a GC. 0 autor, por meio de estudo de softwares, consolida uma taxonomia contendo 8 tipologias de ferramentas tecnológicas de apoio a GC. Tais tipos são relacionados aos processos de geração, codificação e transferência, expostos por Davenport e Prusak (1998), com o intuito de apontar quais desses processos são beneficiados com a 
utilização das ferramentas. As tipologias propostas por Carvalho (2000) são elucidadas a seguir.

Ferramentas voltadas para a intranet: devido à disponibilização da intranet ser em plataforma web, a apresentação do conhecimento na mesma facilita o acesso e seu entendimento tendo em vista que esta plataforma (internet) é difundida na atualidade. Sendo assim, a intranet surge como um meio de veiculação das informaç̧ões e conhecimento da empresa para os funcionários (BENNET, 1997) através da junção de conhecimento de sistemas isolados em um meio único e centralizador, sendo um espaço propício para compartilhamento de informações dinâmicas (DAVENPORT; PRUSAK, 1998). Identifica-se que estas ferramentas propiciam um ambiente favorável a evidenciação do conhecimento (PROBST; RAUB; ROMHARDT, 2002), facilitando a integração, armazenamento, acesso, codificação e transferência do conhecimento (PEREIRA, 2002; STENMARK, 2002), mas não possuem grande ação sobre a geração deste (CARVALHO, 2000).

Sistemas de GED (Gerenciamento Eletrônico de Documentos) surgem com o intuito de tornar acessíveis documentos antigos da organização para melhoria da competitividade, logo, torna-se um repositório de documentos do conhecimento explícito (ORLOWSKA, 1997; DAVENPORT; PRUSAK, 1998; CAVALCANTI; BALCEIRO; GOMES, 2002; MACHADO, 2002) tendo em vista um vasto conhecimento armazenado em documentos eletrônicos (manuais, documentação de processos, entre outros). Devido à característica de ser um repositório do conhecimento, esta tecnologia atua fortemente na codificação e transferência do conhecimento (CARVALHO, 2000).

Sistema de groupware, em sua essência, é uma ferramenta que possibilita o trabalho em equipe de pessoas distantes fisicamente (BOCK; MARCA, 1995; CRUZ, 1998; CANDOTTI; HOPPEN, 1999; TEIXEIRA FILHO, 2001; PEREIRA, 2002). Comumente utilizam-se ferramentas que atuam sobre este conceito como correio eletrônico, videoconferência, dentre outras. Devido à característica da informalidade, esta ferramenta propicia a geração do conhecimento, codificação e transferência do conhecimento (CARVALHO, 2000).

Sistemas de workflow são sistemas que atuam na execução do fluxo de informações dos processos definidos da organização (BOCK; MARCA, 1995; CRUZ, 1998). Em um processo de contratação de seguros, por exemplo, a proposta é enviada e passa pela aprovação dos setores de risco, financeiro, entre outros. Estes setores tomam conhecimento da proposta e atribuem conhecimento para a próxima etapa do processo. Sendo assim, sistemas de workflow são sistemas que auxiliam na codificação e explicitação do conhecimento embutido nos processos (CARVALHO, 2000).

Sistemas para construção de bases inteligentes de conhecimento utilizam da tecnologia de inteligência artificial, que gera o conhecimento através de fatos e conhecimentos armazenados (CARVALHO, 2000). Sendo assim, trata-se de uma tecnologia que atua na geração, codificação e transferência do conhecimento (CARVALHO, 2000).

Business Intelligence (BI) permite às empresas a antecipação de tendências e identificação de informações importantes para o negócio por meio de análise em grandes volumes de dados. Atuam na conversão do conhecimento explícito para explícito (conversão por combinação), codificação e transferência (CARVALHO, 2000).

Sistemas de mapas de conhecimento tem por objetivo relacionar o conhecimento de indivíduos da organização e disponibilizar esta informação para os demais indivíduos (DAVENPORT; PRUSAK, 1998), indicando onde o conhecimento se encontra na firma (VALENTIM, 2013). Trata-se de uma ferramenta que tem o intuito de relacionar informações e pessoas, promovendo o contato e, ou, o encontro entre indivíduos para que ocorra a criação do conhecimento (CARVALHO, 2000; PROBST; RAUB; ROMHARDT, 2002).

Ferramentas de Apoio à Inovação são sistemas que disponibilizam o conhecimento explícito e possibilitam a troca de conhecimento tácito promovendo assim a geração do conhecimento, amparando a criatividade organizacional através da inovação (CARVALHO, 2000). Comumente é representado por bases de conhecimento interna a organização e fóruns de discussão. Promovem a geração, codificação e transferência do conhecimento (CARVALHO, 2000).

Considerando a complexidade de gerir o conhecimento, torna-se indispensável a utilização de ferramentas tecnológicas de apoio a esta gestão, logo as tecnologias aliadas a informação são parceiras indispensáveis nos processos de GC. Feres (2015) salienta que a tecnologia é imprescindível para gerir toda a gama de informação das organizações, e apoia a construção do conhecimento individual e organizacional. Davenport e Prusak (1998, p. 149) 
afirmam que "a gestão do conhecimento é muito mais que tecnologia, mas a tecnologia certamente faz parte da gestão do conhecimento", salientando a importância da tecnologia neste processo.

\section{PROCEDIMENTOS METODOLÓGICOS}

Devido ao fato desta pesquisa abordar um estudo de caso em uma empresa, sob a ótica da gestão do conhecimento e ferramentas tecnológicas de apoio, através do uso da técnica de entrevista aberta, este estudo caracteriza-se como descritivo com abordagem qualitativa.

Para Cervo, Bervian e Da Silva (2007) e Mascarenhas (2012), a pesquisa descritiva visa descrever o processo realizado, para que seja possível identificar e visualizar o que o pesquisador observou e como chegou às conclusões. A abordagem qualitativa, segundo Denzin e Lincoln (2011), constitui em práticas interpretativas através de representações como entrevistas e conversas.

A entrevista aberta consiste na apresentação do tema e questionamento pelo entrevistador seguido de arguição do entrevistado em uma conversação informal (BONI; QUARESMA, 2005). Este método é aplicável a esta proposição uma vez que o intuito é obter o maior número possível de informações e detalhamento sobre o assunto (MINAYO, 1993).

0 estudo de caso tem por característica investigar um fenômeno em um contexto real tornando o objeto de estudo mais claro e ilustrativo (MARTINS; THEÓPHILO, 2009), sendo a Empresa X o objeto deste estudo, cujo a coleta de dados foi realizada através de entrevista aberta a 1 diretor, 2 analistas de sistemas e 3 desenvolvedores de software, com duração de 1 hora e 15 minutos com todos os envolvidos.

A análise dos dados se vale do exame do conteúdo obtido pelas entrevistas, sendo proposta taxonomia de categorias, e subcategorias, com o intuito de identificar características da GC na Empresa X. Tais categorias orientaram os questionamentos realizados no processo de entrevista.

Quadro 1: Categorias, e subcategorias, de análise dos dados.

\begin{tabular}{ll}
\hline Categoria & Descrição \\
\hline Gestão do Conhecimento & Plano de Gestão do Conhecimento \\
\cline { 2 - 2 } & Objetivos e Metas \\
\hline Ferramentas de Apoio a Gestão do Conhecimento & Identificação de Ferramentas \\
\hline
\end{tabular}

Fonte: Dados da pesquisa.

A Empresa X, apresentada sob este nome para preservar sua identidade, foi fundada em 1996 e atua especificamente no mercado de tecnologia da informação através do desenvolvimento de sistemas personalizados com o objetivo suprir necessidades de seus clientes. A firma é classificada como empresa de médio porte, segundo critérios de número de empregados do SEBRAE (2015), pois possui cerca de 150 colaboradores, uma carteira com mais de 40 clientes e define sua meta principal como sendo a "satisfação de seus clientes".

\section{RESULTADOS DA PESQUISA}

\subsection{Dados da entrevista}

1. Nesta seção são apresentados os questionamentos realizados durante a

entrevista na Empresa X. A entrevista foi orientada pelas categorias expostas no

Quadro 1, com o intuito de identificar características da gestão do conhecimento quanto ao seu planejamento e orientação aos objetivos e metas organizacionais e tecnologias utilizadas na empresa para a promoção da GC.

\subsubsection{Gestão do Conhecimento}


Nonaka e Takeuchi (1997) defendem que na era do conhecimento, as empresas devem criar o conhecimento através da obtenção deste de fontes internas e externas à organização e armazená-lo para que seja possível sua utilização.

Tão importante quanto criar o conhecimento é promover a gestão deste para que o mesmo mantenha-se dentro da organização. A implantação da GC dentro de uma organização necessita de um plano de ação, ou plano de gestão do conhecimento (BATISTA, 2012), para que seja possível identificar a evolução da implantação e alcance dos objetivos esperados.

Desta forma, esta seção objetivou indagar à diretoria quanto a implantação da GC orientada aos objetivos e metas da organização.

\subsubsection{Plano de Gestão do Conhecimento}

Buscou-se verificar a existência de uma GC, através da identificação de um plano de gestão do conhecimento. 0 diretor foi indagado pela seguinte questão: "Sr. Diretor, é notório que a Empresa X é uma empresa sólida no mercado de tecnologia onde está presente a mais de 10 anos. $O$ conhecimento adquirido ao longo destes anos sobre tecnologias, concorrentes $e$ mercado possivelmente propicia a Empresa X um apoio à tomada de decisões sobre a estratégia da organização. Existe um Plano de Gestão deste Conhecimento dentro a Empresa X?".

Em resposta ao questionamento realizado, o Diretor esclarece: "Ao decidirmos as estratégias da empresa, para o aumento de carteira de clientes, por exemplo, nos apoiamos sobre metodologias da administração como a análise FOFA (Força, Oportunidade, Fraqueza, Ameaça) entre outras; posteriormente documentamos nossas decisões e construímos nossa estratégia e um plano de ação para alcançar os objetivos. $O$ conhecimento que obtemos fica conosco e posteriormente é exposto nas reuniões e documentados em atas. Posso dizer que não temos um plano de gestão de conhecimento definido e sim um armazenamento de nosso conhecimento por meio de documentos e ferramentas como o Notes, que utilizamos para agendar reuniões e enviar as atas".

Em análise sobre a afirmação da diretoria, observa-se a inexistência de um plano adotado para implantação da GC, conforme fase inicial da GC proposta por Batista (2012), mas é apresentada uma gestão das informações e conhecimento, definida como "[...] armazenamento [...] por meio de documentos e ferramentas como Notes, [...]".

A GC, para ser realizada, precisa ser construída sobre a infra-estrutura de ferramentas de tecnologia da informação, que são a base para realizar a captura, estruturação e transferência do conhecimento, conforme recomendado por Davenport e Prusak (1998).

A Empresa X realiza a captura do conhecimento, por meio de reuniões e o organiza por meio de atas armazenadas na ferramenta Lótus Notes, sendo sua transferência através do envio de tais documentos pela própria ferramenta Lótus Notes. Observa-se a existência de um armazenamento do conhecimento pela Empresa X.

\subsubsection{Objetivos e Metas}

A existência da GC em uma organização, alavancada por ferramentas tecnológicas, apoia a tomada de decisões (VELENTIM et al, 2003) para alcance dos objetivos da empresa. Consequentemente, o alcance deste objetivo torna-se um diferencial obtido pela utilização do conhecimento, uma vez a empresa realiza uma ação que oferece um diferencial aos seus clientes.

Após a identificação da inexistência de uma GC, mas sim de um armazenamento do conhecimento, o Diretor da Empresa $X$ foi questionado quanto aos objetivos e metas esperados pela firma de acordo com o "[...] armazenamento [...] conhecimento por meio de documentos e ferramentas como Notes, [...]".

O entrevistador promoveu o questionamento ao diretor: "A minha próxima pergunta seria em relação às metas e objetivos a serem alcançadas pela organização através do plano de gestão do conhecimento, mas como o Sr. informou a inexistência de um plano, como a empresa identifica o alcance dos objetivos (sucesso e insucesso) de suas estratégias para que melhore no planejamento seguinte?".

Em resposta ao questionamento realizado, o diretor da Empresa X relatou: "Medimos o sucesso / insucesso por período, de acordo com a meta a ser alcançada. Por exemplo, se queremos aumentar a nossa carteira de clientes em $20 \%$ no próximo ano medimos mensalmente o crescimento da carteira de clientes e as ações adotadas para tal crescimento. 
Ao final dos meses temos as ações realizadas que nos impulsionou a atingir ou não a meta, então revisamos nossas ações para o próximo mês / ano".

A Empresa $\mathrm{X}$ realiza um acesso as informações e conhecimentos armazenados e estes subsidiam suas ações futuras tendo em vista a realização de acompanhamento e estudos sobre as metas e objetivos traçados.

Observa-se que o armazenamento do conhecimento provê a Empresa $X$ apoio à tomada de decisões uma vez que este conhecimento é acessado e utilizado pela organização com o intuito de identificar a causa do sucesso e insucesso do atingimento de suas metas: "[...] Ao final dos meses, temos as ações realizadas que nos impulsionou a atingir ou não a meta, então revisamos nossas ações para o próximo mês / ano".

\subsubsection{Ferramentas de Apoio a Gestão do Conhecimento}

Nesta seção serão apresentados os questionamentos realizados aos entrevistados para obter informações acerca das ferramentas tecnológicas utilizadas na empresa com o intuito de confrontar estas tecnologias com a tipologia de ferramentas para a GC, apresentada por Carvalho (2000).

\subsubsection{Identificação de Ferramentas Utilizadas na Empresa $X$}

Com o intuito de identificar os softwares utilizados pela empresa e promover relação das tecnologias com a tipologia proposta por Carvalho (2000), foi solicitado aos entrevistados a relação destes softwares: "Solicitei anteriormente a esta reunião que fosse levantado os softwares utilizados pela empresa para que eu possa entender a utilização dos mesmos pela organização. A seguir, gostaria que me informassem com qual objetivo utilizam cada um destes, caso utilizem"

O Diretor informou que embora seja difícil levantar todos os softwares utilizados pela Empresa X, foram relacionados $60 \%$ a $70 \%$ dos softwares mais utilizados no cotidiano da organização. Em momento seguinte, foi identificada a utilização destes softwares com todos os presentes gerando o Quadro 2.

Quadro 2: Tipos de Softwares e uso na Empresa X.

\begin{tabular}{ll}
\hline Software adotado & Uso do software \\
\hline Acrobat Reader & Utilizado para geração de pdf's e leitura dos mesmos. \\
Citrix Metaframe & Utilizado para conexão remota. \\
Corel Draw & Desenvolvimento de design de sistemas. \\
DB2 & Banco de Dados. \\
Dreamweaver & Desenvolvimento de design de sistemas e desenvolvimento de sites. \\
Editor de Comandos & Utilizado para "rodar" querys no banco de dados DB2. \\
Microsoft Excel & No dia-a-dia da organização em diversas finalidades como armazenar \\
& dados dos funcionários (endereço, contatos, experiências, entre outros) até \\
& planilha de controle de horas. \\
Fireworks: & Desenvolvimento de design de sistemas. \\
IBM Plenus & Gestão do Mainframe da IBM. \\
Internet & Pesquisa. \\
Lótus Notes & Criação de atividades e tarefas para desenvolvimento por outras áreas, \\
& envio de e-mails, conversação instantânea. Basicamente toda \\
& documentação da empresa é utilizada, de alguma forma, nesta ferramenta. \\
Photoshop: & Desenvolvimento de design de sistemas. \\
Power Point & Apresentação de slides em reuniões. \\
Project & Gestão de Projetos. \\
Source Safe & Gestão de Configuração. \\
SQL Server & Banco de Dados. \\
System Architect & Criação de especificação UML e diagramas de banco de dados. \\
Visual Basic & Programação de sistemas. \\
Visual Studio & Programação de sistemas. \\
Webservice Studio & Programação de sistemas. \\
Winrar e Winzip & Compactador de Arquivos. \\
Word & Criação de documentos diversos como atas de reuniões e casos de uso. \\
\hline Fonte: Dados &
\end{tabular}

Fonte: Dados da pesquisa.

Diante da relação diversa de sistemas e usos levantados anteriormente, os entrevistados foram questionados quanto ao real uso dos softwares no desenvolvimento de suas atividades. Este questionamento objetivou filtrar os sistemas que, de fato, possuem 
relevância nas atividades cotidianas da organização a fim de promover uma análise mais objetiva.

Desta forma, foi realizado o seguinte questionamento ao diretor, analistas e desenvolvedores de sistemas quanto à utilização das ferramentas relatadas anteriormente: "De forma macro, gostaria que descrevessem a utilização das ferramentas citadas anteriormente no cotidiano de suas atividades. Obviamente nem todas as ferramentas são utilizadas ou algumas são utilizadas esporadicamente logo, peço que destaquem as de utilização rotineira".

Posteriormente ao questionamento, foi obtido os relatos de cada entrevistado.

Diretor: "Utilizo o Notes e Word para armazenar as informações / conhecimentos sempre que tenho reuniões de acompanhamento dos nossos objetivos, além de outras atividades que demandam documentação. A diretoria identifica o propósito de utilização do Notes e Word como uma forma de documentar as nossas ações para que possamos consultar as mesmas novamente".

Analista 1: "Utilizo o Word e SSafe com uma frequência diária, pois temos que criar diversas especificações e diagramas, o Notes para enviar e-mails, agendar reuniões e contatar os clientes através da ferramenta SameTime e vejo que é de extrema importância para que eu possa acompanhar o andamento do sistema registrando a evolução do mesmo".

Analista 2: "Também utilizo diariamente as ferramentas que foram ditas pelo Analista 1 e, além da importância que ele mencionou, percebo que sempre que preciso de alguma informação, ou de algum referencial para minhas atividades, tenho onde procurar pois tudo fica armazenado no SSafe".

Desenvolvedor 1: "Além do envio de e-mail do Notes, que é a ferramenta utilizada por todos para enviar e-mail na empresa e para acompanhar o desenvolvimento das atividades, $e$ do SSafe para armazenar o código, também uso o SameTime e a Internet para tirar dúvidas diariamente em relação à programação. Net e não vejo como eu conseguiria programar o que me é passado sem a utilização destas ferramentas".

Desenvolvedor 2: "Mesma coisa do Desenvolvedor 1".

Desenvolvedor 3: "Também concordo com o Desenvolvedor 1".

$\mathrm{O}$ diretor da Empresa $\mathrm{X}$ manifestou que existe na empresa uma planilha Excel que relaciona os funcionários com o conhecimento que os mesmos possuem. Informou também que esta planilha fica disponível em uma pasta pública onde todos possuem acesso.

A partir dos relatos de usos dos sistemas, foram identificadas características das ferramentas de maior utilização no cotidiano dos entrevistados promovendo a relação de cinco tecnologias de maior intensidade de uso nas atividades dos envolvidos: internet, Lótus Notes, Visual SSafe, .NET (Microsoft Visual Studio) e Microsoft Word.

Quadro 3: Visão do usuário quanto a Ferramenta INTERNET.

\begin{tabular}{lll}
\hline Usuário & Objetivo Percebido & Frequência de Utilização \\
\hline Diretor & Não se aplica & Não se aplica \\
Analistas & Não se aplica & Não se aplica \\
Desenvolvedor & Buscar conhecimento & Diária \\
\hline
\end{tabular}

Fonte: Dados da pesquisa.

A internet possui principal uso, segundo relatos dos entrevistados, pelos desenvolvedores para a solução de problemas relacionados a programação dos sistemas, sendo seu uso diário. 0 diretor e analistas não salientaram o uso desta tecnologia nas atividades desempenhadas.

Quadro 4: Visão do Usuário quanto a Ferramenta LÓTUS NOTES.

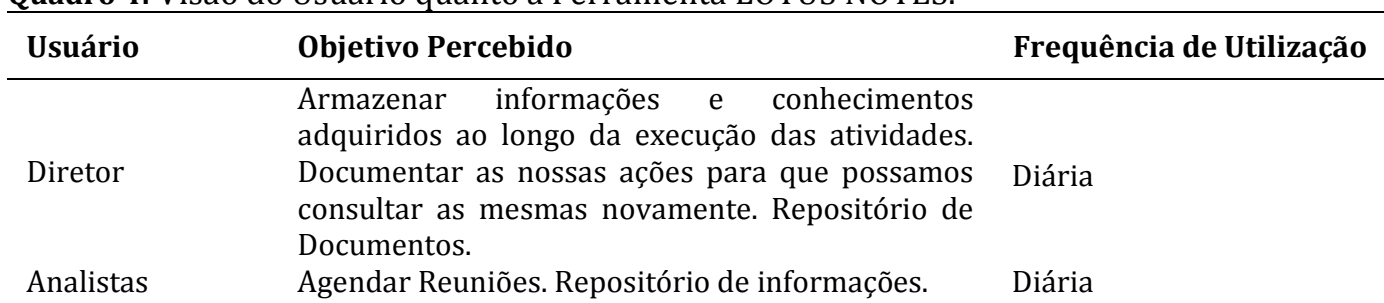


Fonte: Dados da pesquisa.

A ferramenta Lótus Notes é de uso de todos os envolvidos e aborda diversas ações, sendo seu uso diário na organização. Esta ferramenta suporta diversos aplicativos como correio eletrônico, agenda, conversas instantâneas, dentre outras além de poder ser customizada trazendo dinamicidade e integração entre sistemas da firma.

Quadro 5: Visão do Usuário quanto a Ferramenta VISUAL SSAFE.

\begin{tabular}{|c|c|c|}
\hline Usuário & Objetivo Percebido & Frequência de Utilização \\
\hline Diretor & Não se aplica & Não se aplica \\
\hline Analistas & $\begin{array}{l}\text { Armazenar documentos dos sistemas. } \\
\text { Repositório de Documentos. }\end{array}$ & Diária \\
\hline Desenvolvedor & $\begin{array}{l}\text { Armazenar códigos dos sistemas. Repositório } \\
\text { de Documentos. }\end{array}$ & Diária \\
\hline
\end{tabular}

Fonte: Dados da pesquisa.

O Visual SSafe é utilizado pelos analistas e desenvolvedores de sistemas diariamente como um repositório de documentos e códigos de programas. Comumente as organizações de tecnologia da informação utilizam ferramentas de versionamento, uma vez que necessitam armazenar versões de artefatos sistêmicos.

Quadro 6: Visão do Usuário quanto a Ferramenta VISUAL STUDIO.

\begin{tabular}{lll}
\hline Usuário & Objetivo Percebido & Frequência de Utilização \\
\hline Diretor & Não se aplica & Não se aplica \\
Analistas & Não se aplica & Não se aplica \\
Desenvolvedor & Codificar os sistemas & Diária \\
\hline
\end{tabular}

Fonte: Dados da pesquisa.

O Microsoft Visual Studio é uma plataforma de desenvolvimento de sistemas utilizada pelos desenvolvedores, diariamente, para este fim na Empresa X. Como se trata de uma tecnologia para um fim específico, justifica-se o não uso pelos demais perfis de usuários entrevistados.

Quadro 7: Visão do Usuário quanto a Ferramenta MICROSOFT WORD.

\begin{tabular}{|c|c|c|}
\hline Usuário & Objetivo Percebido & Frequência de Utilização \\
\hline Diretor & $\begin{array}{l}\text { Armazenar as informações e conhecimentos } \\
\text { adquiridos ao longo da execução das atividades do } \\
\text { dia. }\end{array}$ & Diária \\
\hline Analistas & Armazenar informações obtidas nas reuniões & Diária \\
\hline Desenvolvedor & Não se aplica & Não se aplica \\
\hline
\end{tabular}

Fonte: Dados da pesquisa.

O Microsoft Word, enquanto uma tecnologia de edição eletrônica textos, é utilizado pelos analistas e diretor com o intuito de documentar informações sobre reuniões e atividades cotidianas, respectivamente. Os desenvolvedores não relatam o uso desta tecnologia.

Quadro 8: Visão do Usuário quanto a Ferramenta SAMETIME (LÓTUS NOTES).

\begin{tabular}{lll}
\hline Usuário & Objetivo Percebido & \multicolumn{1}{c}{ Frequência de Utilização } \\
\hline Diretor & Não se aplica & Não se aplica \\
Analistas & Contatar clientes. & Diária \\
Desenvolvedor & Sanar Dúvidas & Diária \\
\hline
\end{tabular}

Fonte: Dados da pesquisa. 
O SameTime é um aplicativo do Lótus Notes utilizado para conversação instantânea e seu uso é diário para os analistas e desenvolvedores da organização, sendo um canal de contato com clientes pelos analistas e tira dúvidas para os desenvolvedores.

\subsection{Análise dos Resultados}

A partir da identificação das cinco ferramentas utilizadas na Empresa X, sendo internet, Lótus Notes, Visual SSafe, .NET (Microsoft Visual Studio) e Microsoft Word, estas foram analisadas quanto ao enquadramento na tipologia apresentada por Carvalho (2000), conforme Quadro 9.

Quadro 9: Tipologia de Ferramentas de Gestão do Conhecimeto Adotadas pela Empresa X.

\begin{tabular}{ll}
\hline Tipologia de Ferramentas de GC & Ferramenta Adotada pela Empresa $\boldsymbol{X}$ \\
\hline Ferramentas voltadas para a Intranet & Não se aplica \\
Sistemas de GED (Gerenciamento Eletrônico de & Visual SSafe \\
$\begin{array}{l}\text { Documentos) } \\
\text { Sistema de Groupware }\end{array}$ & Lótus Notes (SameTime) \\
Sistemas de Workflow & Lótus Notes \\
Sistemas para Construção de Bases Inteligentes de & Não se aplica \\
Conhecimento & Não se aplica \\
Business Intelligence (BI) & Excel \\
Sistemas de Mapas de Conhecimento & Não se aplica \\
Ferramentas de Apoio à Inovação &
\end{tabular}

Fonte: Dados da pesquisa.

Em análise das ferramentas apresentadas pela Empresa $\mathrm{X}$ identificou-se que as ferramentas Visual SSafe, SameTime, Lótus Notes e Microsoft Excel enquadram-se nas tipologias sistemas de GED, groupware, workflow e mapas de conhecimento, respectivamente.

A ferramenta Visual SSafe condiz com sistemas de GED por atuar como um repositório de documentos do conhecimento explícito (ORLOWSKA, 1997; DAVENPORT; PRUSAK, 1998; CAVALCANTI; BALCEIRO; GOMES, 2002; MACHADO, 2002), relacionados a documentos da organização e códigos sistêmicos

O SameTime é uma ferramenta de uso para conversação instantânea na Empresa X, sendo um sistema classificado na taxonomia de groupware por possibilitar o trabalho em equipe de pessoas distantes fisicamente (BOCK; MARCA, 1995; CRUZ, 1998; CANDOTTI; HOPPEN, 1999; TEIXEIRA FILHO, 2001; PEREIRA, 2002).

A ferramenta Lótus Notes é relacionada aos sistemas de workflow pelo ao apoio ao fluxo de informações dos processos definidos da organização (BOCK; MARCA, 1995; CRUZ, 1998), utilizada na Empresa X para o tramite de informações por correio eletrônico, agenda, conversas instantâneas, dentre outras.

O Microsoft Excel alicerça a taxonomia de sistemas de mapas de conhecimento por relacionar informações de pessoas (endereço, contatos, experiências, entre outros), promovendo o contato e, ou, o encontro entre indivíduos para que ocorra a criação do conhecimento organizacional (CARVALHO, 2000; PROBST; RAUB; ROMHARDT, 2002; VALENTIM, 2013).

As demais tipologias não são exploradas pela empresa sobre o ponto de vista da análise realizada sobre as ferramentas apresentadas pelos entrevistados da Empresa X. Esta análise não considera características das ferramentas, e sim o enquadramento das mesmas nas tipologias de Carvalho (2000), de acordo com sua utilização na Empresa X.

Para Davenport e Prusak (1998, p. 61), a gestão do conhecimento, para ser realizada, precisa ser construída sobre a infra-estrutura de ferramentas de tecnologia da informação, que são a base para realizar a captura, estruturação e transferência do conhecimento. As ferramentas tecnológicas de apoio a gestão do conhecimento são imprescindíveis para a realização desta gestão. Sem estas o conhecimento torna-se inacessível ou de difícil acesso, dificultando a transferência do conhecimento dentro da organização.

Observa-se que as ferramentas utilizadas pela empresa, que se enquadram na tipologia de Carvalho (2000), atendem a 4 (quatro) das 8 (oito) tipologias apresentadas, mas sem uma GC as mesmas são subutilizadas para este propósito. A inexistência de um Plano de 
GC pela Empresa X, torna possível inferir a ausência de uma gestão direcionada ao conhecimento.

Nonaka e Takeuchi (1997, p. 4) defendem que na era do conhecimento, as empresas devem criar o conhecimento através da obtenção deste de fontes internas e externas à organização e armazená-lo para que seja possível sua utilização.

Embora a Empresa X não possua uma GC formalizada, é possível inferir que a mesma realiza, de forma simplória, a obtenção do conhecimento de fontes internas da empresa, como é feito através do acompanhamento das metas de aumento da carteira de clientes, e o armazenamento destes em arquivos eletrônicos através de ferramentas como Microsoft Word e Microsoft SSafe. O armazenamento do conhecimento adquirido é utilizado em algumas atividades, e desta forma promovem um apoio à tomada de decisão que impulsiona a organização a manter-se competitiva no mercado de atuação.

Não é mencionada a mensuração do conhecimento dentro da empresa logo, como não existem ações para metrificar este ativo observa-se a ausência de ações para promover sua evolução.

Vê-se que de forma inconsciente, é percebido pela diretoria que se a meta foi atingida o conhecimento gerido pela organização obteve sucesso, mas caso a meta não tenha sido atingida, o conhecimento não é avaliado, mas sim as ações que a nortearam ao insucesso, desconsiderando uma análise para evolução do conhecimento organizacional e de sua gestão.

De acordo com o estudo realizado sobre a Empresa X quanto a GC e as tipologias de apresentadas por Carvalho (2000), a implantação de algumas tipologias, junto à construção de um planejamento prévio, são sugestões de melhorias identificadas durante o estudo.

Quadro 10: Propostas de Melhoria

\begin{tabular}{|c|c|c|}
\hline $\begin{array}{l}\text { Tipologia de } \\
\text { Ferramentas de GC }\end{array}$ & Ponto de Melhoria & Descrição da Melhoria \\
\hline $\begin{array}{l}\text { Ferramentas } \\
\text { voltadas para a } \\
\text { Intranet }\end{array}$ & Criação de Intranet & $\begin{array}{l}\text { Implementação de uma intranet para centralização } \\
\text { das informações internas da organização e } \\
\text { disponibilização de modelos de documentos como } \\
\text { especificação de softwares, atas, dentre outros. }\end{array}$ \\
\hline
\end{tabular}

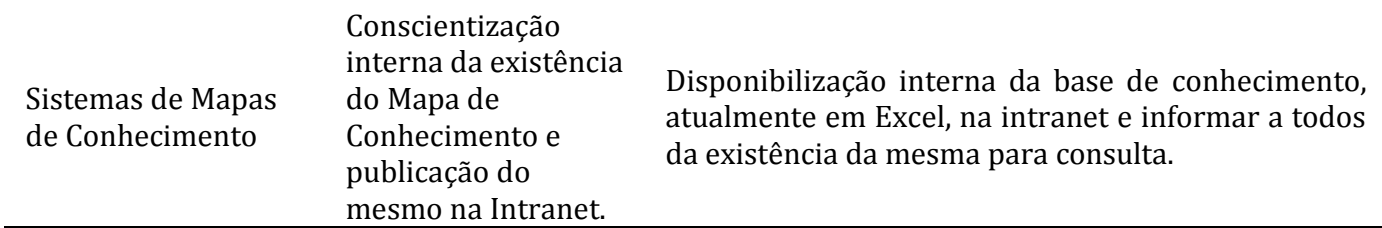

Fonte: Próprio autor.

A Quadro 10 apresenta sugestões de implementação de tipologias juntamente com o ponto de melhoria a ser considerado com sua implementação, identificado através do estudo de caso sobre a Empresa X.

\section{CONSIDERAÇ̃̃ES FINAIS}

A implementação destas tipologias gera custo a organização, entretanto na sociedade do conhecimento é necessário considerar este investimento, que pode ser minimizado através do uso de ferramentas gratuitas que implementam estas tipologias e que atendam às necessidades da organização.

A proposta de um estudo de caso sobre GC em uma empresa de tecnologia da informação tem por intuito fomentar um olhar do uso de tecnologias em empresas atuantes neste setor, trazendo assim uma reflexão paradoxal de como empresas de base tecnológica utilizam a tecnologia para a GC.

Atividades exercidas pelos colaboradores da organização podem ser aprimoradas com a implementação da GC como, por exemplo, a criação de uma intranet para prover interação entre os colaboradores e potencializar a geração e compartilhamento do conhecimento explícito, e a disponibilização do mapa de conhecimento, já existente na organização, entre os colaboradores para que seja possível direcionar os colaboradores a um especialista, otimizando o tempo de pesquisa destes dentro da organização. 
Mesmo sem uma GC formalizada, a empresa se mantém competitiva uma vez que realiza estudo sobre as metas atingidas, e não atingidas, ao longo dos meses com o intuído de adequar o Planejamento Estratégico. Obviamente esta não é a única atividade desempenhada que a torna sólida no mercado, embora tenha sido a única identificada durante a entrevista realizada junto à organização.

Esta pesquisa não realiza uma análise de utilização das ferramentas adotadas pela organização, mas sim uma identificação da percepção do usuário de tais ferramentas quanto a sua utilidade, logo o objetivo não é propor uma evolução, ou substituição, das ferramentas e sim a implementação de tipologias que podem trazer um diferencial à empresa.

Como sugestão de pesquisas futuras, sugere-se aplicar este estudo em outras empresas de base tecnológica e de diferentes portes para prover comparação entre os estudos, além da aplicação de estudos longitudinal para identificar a evolução da organização após determinado período de tempo.

\section{REFERÊNCIAS}

BARNEY, J. B.; HESTERLY, W. S. Administração estratégica e vantagem competitiva. São Paulo: Pearson Prentice Hall, 2007.

BASTOS, Carlos Alberto Malcher; BRUNO, Ana Cristina Martins; REZENDE, Luiziana Silveira de. Gestão da informação e do conhecimento: proposição de um modelo integrador a partir da identificação e gestão dos bens de informação. In: CIANCONI, Regina de Barros; CORDEIRO, Rosa Inês Novais; ALMEIDA, Carlos Henrique Marcondes de (org.). Gestão do conhecimento, da informação e de documentos em contextos informacionais. Niterói: PPGCI/UFF, 2013. p. 27-46.

BATISTA, Fábio Ferreira. Modelo de gestão do conhecimento para a administração pública brasileira: como implementar a gestão do conhecimento para produzir resultados em benefício do cidadão. Brasília, DF: Instituto de Pesquisa Econômica Aplicada (Ipea), 2012. Disponível em: <http://repositorio.ipea.gov.br/handle/11058/754>. Acesso em 15 jan. 2015.

BENETT, Gordon. Intranets: como implantar com sucesso na sua empresa. Rio de Janeiro: Campus, 1997.

BOCK, Geoffrey; MARCA, David. Designing Groupware. Nova Iorque: McGraw-Hill, 1995. BONI, Valdete; QUARESMA, Sílvia Jurema. Aprendendo a entrevistar: como fazer entrevistas em Ciências Sociais. Em Tese, v. 2, n. 1, p. 68-80, 2005.

CANDOTTI, Clarissa Tarragô; HOPPEN, Norberto. Reunião virtual e o uso de groupware uma nova possibilidade de realizar trabalho em grupo. In: ENCONTRO ANPAD, 23., Foz do Iguaçu/RS, 1999. Anais... Foz do Iguaçu: ANAPAD, 19 a 22 de setembro de 1999.

CARVAlHO, Rodrigo Baroni de. Aplicações de Softwares de Gestão do Conhecimento: Tipologia e Usos. 2000. 144 f. Dissertação (Mestrado em Ciência da Informação). Programa de Pós-graduação em Ciência da Informação, UFMG, Belo Horizonte; Disponível em: <http://www.bibliotecadigital.ufmg.br/dspace/bitstream/handle/1843/LHLS69XQRP/mest rado_rodrigo_baroni_de_carvalho.pdf?sequenc=1>. Acesso em: 1 jan 2014.

CAVALCANTI, Marcos do Couto Bezerra; BALCEIRO, Raquel Borba; GOMES, Elisabeth Braz Pereira. A Prática da Gestão de Conhecimento nas Empresas Públicas. Rio de Janeiro: Epapers. 2002.

CERVO, Amado Luiz; BERVIAN, Pedro Alcino; DA SILVA, Roberto. Metodologia Científica. 6. ed. São Paulo: Pearson Prentice Hall, 2007.

CHOO, C. W. A organização do conhecimento: como as organizações usam a informação para criar significado, construir conhecimento e tomar decisões. São Paulo: Senac, 2003. 
CORRÊA, Fábio. A gestão do conhecimento aplicada ao setor de tecnologia da informação. 2014. 175 f. Dissertação (Mestrado em Gestão de Sistemas de Informação e do Conhecimento) - Faculdade de Ciências Empresariais, Universidade FUMEC, Belo Horizonte, 2014. Disponível em: <http://www.fumec.br/revistas/sigc/article/download/2553/1624>. Acesso em: 13 maio 2015.

CRUZ, Tadeu. Workflow: A tecnologia que vai revolucionar processos. São Paulo: Atlas, 1998.

DAVENPORT, Thomas H; PRUSAK, Laurence. Conhecimento empresarial: como as organizações gerenciam o seu capital intelectual. 11. ed. Rio de Janeiro: Campus, 1998.

DENZIN, Norman K.; LINCOLN, Yvonna S. The SAGE handbook of qualitative research Sage, 2011.

DRUCKER, Peter. 0 melhor de Peter Drucker: homem, sociedade, administração. São Paulo: Nobel, 1998.

FERES, Glória Georges. Competência em informação: interface entre as redes de conhecimento, criatividade e inovação. In: BELLUZO, Regina Célia Baptista; FERES, Glória Geroges; VALENTIM, Marta Lígia Pomim (org.). Redes de conhecimento e competência em informação: interfaces da gestão, mediação e uso da informação. Rio de Janeiro: Interciência, 2015. p. 215-245.

FONTANILLAS, Carlos Navarro; CRUZ, Eduardo Picanço; GONÇALVES, Jaqueline Pimentel de Souza. A gestão do conhecimento e os processos de informações nas organizações: estudo de caso em uma empresa off shore. Sustainable Business International Journal, n. 18, p. 1-28, 2012. Disponível em:

<http://www.sbijournal.uff.br/index.php/sbijournal/article/view/26>. Acesso em: 07 jan. 2016.

KATZENBACH, J.R. e SMITH, D. K. Equipes de alta performance: conceitos, princípios e técnicas para potencializar o desempenho das equipes. Rio de Janeiro: Campus, 2001.

MACHADO, Rogério Brasiliense. Gerenciamento eletrônico de documentos e sua interrelação com a gestão o conhecimento. In: ANGELONI, Maria Terezinha (org.). Organizações do conhecimento: infra-estrutura, pessoas e tecnologia. São Paulo: Saraiva, 2002. p. 196210.

MARTINS, G. A.; THEÓPHILO, C. R. Metodologia da investigação científica para ciências sociais aplicadas. 2. ed. São Paulo: Atlas, 2009.

MASCARENHAS, Sidnei Augusto. Metodologia científica. São Paulo: Pearson Education do Brasil, 2012.

MINAYO, Maria Cecília de Souza. o desafio do conhecimento científico: pesquisa qualitativa em saúde. 2. ed. São Paulo/Rio de Janeiro: Hucitec-Abrasco, 1993.

NEWSTROM, John W.; PIERCE, John L. A Estante do administrador: uma coletânea de leituras obrigatórias. São Paulo: Bookman, 2002.

NEWELL, Sue; SCARBROUGH, Harry; SWAN, Jacky. From global knowledge management to internal electronic fences: contradictory outcomes from intranet development. British Journal of Management, v. 12, n. 2, p. 97-111, 2001. Disponível em: <http://onlinelibrary.wiley.com/doi/10.1111/1467-8551.00188/abstract>. Acesso em: 29 set. 2014.

NONAKA, Ikujiro; TAKEUCHI, Hirotaka. Criação do conhecimento na empresa: como as empresas japonesas geram a dinâmica da inovação. São Paulo: ELSEVIER, 1997. 
ORLOWSKA, Maria E.; SADIQ, Wasim. Applying a Generic Conceptual Workflow Modeling Technique to Document Workflow. In: PROC. AUSTRALIAN DOCUMENT COMPUTING SYMPOSIUM, 2., Melbourne, 1997. Proceedings... Melbourne: [s.n.], April 1997.

PEREIRA, Rita de Cássia Faria. As redes como tecnologia de apoio a gestão do conhecimento. In: ANGELONI, Maria Terezinha (org.). Organizações do conhecimento: infra-estrutura, pessoas e tecnologia. São Paulo: Saraiva, 2002. p. 156-171.

PETERAF, M. A.; BARNEY, J. B. Unraveling the resource-based tangle. Managerial and Decision Economics, 24(4), 309-323, 2003.

PORTER, M. Vantagem competitiva: criando e sustentando um desempenho superior. Rio de Janeiro: Campus, 1990.

PROBST, Gilbert; RAUB, Steffen, ROMHARDT, Kai. Gestão do conhecimento: os elementos construtivos do sucesso. Porto Alegre, Editora Bookman, 2002.

SEBRAE - Serviço de Apoio às Micro e Pequenas Empresa. Critérios de Classificação de Empresas: EI - ME - EPP, 2014. Disponível em: <http://www.sebraesc.com.br/leis/default.asp?vcdtexto=4154>. Acesso em: 9 dez. 2015.

SETZER, Valdemar W. Dado, Informação, Conhecimento e Competência. DataGramaZero Revista de Ciência da Informação - n. zero dez/99

SPENDER, J. C. Gerenciando sistemas de conhecimento. In: FLEURY, Maria Tereza Leme; OLIVEIRA JR, Moacir de Miranda. Gestão estratégica do conhecimento: integrando aprendizagem, conhecimento e competências. São Paulo: Atlas, 2001.

STENMARK, Dick. Information vs. knowledge: The role of intranets in knowledge management. In: ANNUAL HAWAII INTERNATIONAL CONFERENCE ON SYSTEM SCIENCES HICSS, 35., Hilton Waikoloa Village/HW, 2002.. Proceedings... Hilton Waikoloa Village/HW: IEEE, p. 928-937, January 7-10 2002.

SVEIBY, Karl-Erik. A nova riqueza das organizações: Gerenciando e avaliando patrimônios do conhecimento. 5. ed. Rio de Janeiro: Campus, 1998.

TEIXEIRA FILHO, Jayme. Gerenciando conhecimento. 2. ed. Rio de Janeiro: SENAC, 2001.

TERRA, José C. C. Gestão do conhecimento: o grande desafio empresarial. 2. ed. São Paulo: Negócio, 2001.

TERRA, José C. C.; GORDON, Cindy. Portais corporativos. São Paulo: Negócio, 2002.

TOFFLER, Alvin. A terceira onda. São Paulo: Record, 1987.

VALENTIM, Marta Lígia Pomim et al. O processo de inteligência competitiva em organizações. DataGramaZero, Rio de Janeiro, v. 4, n. 3, p. 1-23, 2003.

VALENTIM, Marta Lígia Pomim. A importância do compartilhamento de conhecimento em ambientes empresariais. In: CIANCONI, Regina de Barros; CORDEIRO, Rosa Inês Novais; ALMEIDA, Carlos Henrique Marcondes de (org.). Gestão do conhecimento, da informação e de documentos em contextos informacionais. Niterói: PPGCI/UFF, 2013. p. 59-80.

YOUNG, Ronald. Knowledge Management: tools and techniques manual. Tokyo: Asian Productivity Organization, 2010. Disponível em: <http://www.apo-tokyo.org/00e-books/IS43_KM-Tools_and_Techniques_2010/IS-43_KM-Tools_and_Techniques_2010.pdf >. Acesso em: 23 jan. 2014.

Editores do artigo: Adilson Luiz Pinto, Rafaela Paula Schmitz e Enrique Muriel-Torrado 